dre les plus divers, à interruptions multiples avec souffleur's magnétiques, à condensateurs et à décharge continuc par le moyen d'une résistance élevée. Tout fait espérel que la pratique prouvera leur bon fonctionnement. Il est intéressant de signaler les précautions qui ont été prises pour ćviler toute interruption de service en sectionnant la ligne en plusieurs tronçons, et en la faisant inspecter par des postes permanents de gardiens placés environ lous les 15 kilomètres.

Ces différents postes sont reliés entre eux par une douljle ligne téléphonique, ainsi qu'avec les stations génératrices el réceptrices.

Des appareils de contrôle permettant la mise rapide en service, ou hors service, des differents groupes et la simplicilé des tableaux et de lappareillrge doit tout particulièrement être remarquée, car une unité peut être mise en circuit ou hors circuit par la simple manœuvre diun seul interupteur.

Nous dernns revenir sur un point important que nous navons fail que mentionner, nous voulons parler de la partie de la ligne d'envirn cing kilomètres de longueur lormée de deux câbles souterrains à un seul conducteur.

Co cable constitue une innovation,car. à part le câble triphasć à 27000 volts placé à titre d'essai à Toulon pour le comple de la Société francaise pour l'Exploitation des procedes Thomson-Houston et construit par la maison Groffroy et Delore, nous ne connaissons pas d'exemple de cáble directoment intercalé dan's une ligne de transport de force à tension aussi èlevée.

Dans le cas qui nous occupe, le câble doit résister à une tension normale de 50000 volts entre son âme et la terre, mais il a été éprouvé aux essais a 75000 volis alternatifs correspondant à près de 106000 volts courant continu, soit plus du double de la tension de service.

Le câble a été fourni pal la maison Berthoud-Borel de Lyon.

Afin de diminuer dans la mesire du pocsible tontea les interruptions de service. il a été in-tallé a l'aurivée a L,yon une station transformatrice de secours, composie de moleur's à conra it continu accouples a les genoratrices triphasces, 10000 volts, dépendant elles-mêmes du réseau gênéral alternatif à 30000 volts de la Société Grenobloise de Forcc et Lumière qui pénètre jusqu'à Lyon.

Ces dynamos conjuguées forment des groupes reversibles.

Nous voulons dire par là que chaque groupe peut aussi hien recevoir du courant continu et produire du courant altermatif qu'il peut alssorber du courant alternatif et engendrer du courant continue série.

Des régulateurs doubles sur les unités à courant continu permettent ces deux fonctionnements.

Cette station peut donc, soit livrer du courant alternatif triphase 10000 volts pour divers usages, soit fonctionner comme station génératrice de courant continu série et venir au secours de la station génératrice de Moutiers.

Nous serions incomplets si nous ne mentionnions pas la question du retour par la terre qui a falt l'objet d'intéressantes études, en 1902, sur l'initiative de MM Bergès, et qui ont étê suivies par une commission désignêe par le Comité d'Electricité sous la présidence de M. Harlé.

La possibilité de la marche du système avec un fil mis partiellement ou totalement à la terre a bien été envisagée, mais, pratiquement, il a dù y être renoncé, vu les difficultés de tabler, pour le moment encore, sur ce mode de fonctionnement aussi longtemps qu'il n'aura pas été plus dùment éprouvé, et qu'il n'aura pas été autorisé, car il n'est jas sans entrainer quelques troubles locaux du fait des courants telluriques.

En terminant, c'est peut-être la place ici de rappeler une communication présentée à l'Académie des Sciences en 1881, par M. Cornu, au nom de M. Cabanellas.

Il est intéressant de noter que, déjà à rette époque, M. Cabanellas, comme un précurseur, faisait prévoir le parti qu'on pourrait tirer du fonctionnement des dynamos branchées en série.

Plus tard, en 1886, M. Fontaine soumeltait à l'Académie le résultat de ses expériences pour obtenir des hautes tensions en remplaçant une seule machine receptrice par diverses machines branchées en série.

Ce n'est, cependant, quaprès 1890 que le système reçut une première application importante en Italie, qui permit à $M$. Thury, actuellement ingénieur en chef de la Compagnie de l'Industrie Electrique el Mécanique, de mettre au point. a cette occasion, la question du reglage qu'il a perfec. tionnée depuis et réalisée d'une fnçon que l'on peut dire parfaite dars l'exemple que nous renons d'exposer, car c'est à cette Compagnie qu'est due la construction du maté. riel courant continu série installé à Moutiers et à Lyon.

Nous ne voulons pas tirer de conclusions générales sur les applications que l'on peut faire de ce systeme, car à chaque cas correspond une solution spéciale qui résulte des conditions toujours variables du problème à résoudre, mais nous devons insister sur lintérćt considérable et sur l'avenir que nous croyons réservés a la facilité de constituer en cables souterrains les lignes a courant continu série.

L'essai couronné de succes fait par la Sociète Grenobloise de Force et Lumiere mérite, à ce point de rue tout special, de retenir l'attention.

\section{LA TRACTION ÉLECTRIQUE SUR LES CHEMINS DE FER}

Rapport de M. Auvert, ingénieur principal à la Compagnie P.-L.-M.

Depui- Innstomp-réjà, les Ineénieurs éloctriciens de tous les pays nnt compris que l'électricité pourmut, sous certaines conditions, être avantagreusoncnt uliliséc à la traction sur les chemins de ter.

De nombreuses et patientes recherches, lant théoriques que pratiques, de coùteuses expériences, ont ćté faites, et elles ont été suivies d'un certain nombre d'applications.

Quelques-unes de celles-ci n'ayant pas donné dans la pratique les résultats économiques que leurs promoteurs en attendaient, les adversaires du nouveau mode de traction ont pu, par une généralisation quelque peu hâtive, conclure que l'électricité, excellente pour la traction sur les tram. ways urbains, ou interurbains, et sur les chemins de fer métropolitains, ne pourrait jamais faire une concurrence sérieuse à la vapeur pour la traction sur les grandes lignes.

Une telle conclusion ost d'autant moins justifiée que les mauvais résultats économiques, auxquels je viens de faire allusion, proviennent de ce fait qu'on s'est généralement borné à appliquer aux chemins de fer les procédés de traction couramment employés sur les tramways ou les métropolitains, où les réseaux sont beaucoup moins etendus en longueur, et où la circulation est relativement très régulière.

$\mathrm{Or}^{*}$, si on laisse de côlé les mélropolitains, ớ l'emṕlai de l'électricité présente des avantages spéciaux qui la font prélérer à la vapeur en dehors de toute considération économique, il convient de remarquer que les tracteurs mécaniques pour tramways à vapeur ou à air comprimé laissent généralement beaucoup à désirer, ils sont peu économiques tant au point de vue de la consommation qu'à celui de l'entretien.

Dans ces conditions, la substitution de la traction élec- 
trique (*) à ces procédés de traction surannés a réalisé un grand progrès à tous les points de vue.

Sur les chemins de fer, au contraire, la traction à vapeur est arrivée aujourd'hui à un haut degré de perfection, et les locomotives à vapeur modernes constituent de merveilleux engins, grâce auxquels le prix moyen de la tonne kilomètre est descendu à un taux remarquablement bas, malgré l'accroissement de la vitesse de marche.

Il résulte de ce qui précède que les anciens procédés de traction électrique qui avaient triomphé sans conteste sur les tramways, et dans lesquels on avait cherché avant tout la simplicité et la légèreté, souvent au détriment de l'économie de fonctionnement, ne peuvent lutter avec la traction à vapeur sur les chemins de fer, sauf dans des cas spéciaux où la question d'économie doit être laissée au second plan.

\section{EMPloi du Courant continu}

Jusqu'à ces dernières années, tant en Europe qu'en Américue, on faisait exclusivement usage, sur les tramways électriques, du système de traction à courant continu dont les dispositions caractéristiques sont les suivantes:

Production de l'énergic électrique dans une station centrale, soit directement sous forme de courant continu à la tension de 500 à 600 volts, soit sous forme de courants alternatıfs triphasés à haute tension, transmis par une canalisation spéciale dans des sous-stations ou ces courants triphasés sont transformés en courant continu à la tension de 550 à 600 volts, au moyen de transformateurs statiques et de convertisseurs.

Distribution du courant continu aux véhicules moteurs au moyen d'une canalisation généralement aérienne et d'un contact glissant; cetto canalisation peut d'alleurs, suivant les cas, recevoir des dispositions variées (systèmes à caniveau, systèmes à contacts superficiels, etc.).

Utilisation du courant continu dans des moteurs à excitation série pouvant être groupés de diverses façons, mais toujours réglés au moment des démarrages et changements de vitesse, au moyen de résistances intercalées dans le circuit principal.

Quand on a voulu passer de la traction sur les tramways à la traction sur les chemins de fer, on a tout naturellement cherché à utiliser le même système, et on s'est d'abord contenté d'augmenter considérablenent la puissance des moteurs et les dimensions dos divers accessoires de réglagie et de contrôle.

Quant au conducteur de prise de courant, comme il ne pouvait être maintenu aérien en raison de ses dimensions et de son poids. on l'a constitué à l'aide d'une barre d'acier, généralement un rail, placée le long de la voie sur des supports isolants fixés aux traverses; et de là est venu le nom de système à troisième rail.

En mème temps, on a, dans certains cas, modifié le système usuel de liaison des moteurs aux roues motrices, de manière à atténuer autant que possible les réactions dues aux inégalitéz de la voie, réactions d'autant plus à craindre que les moteurs sont plus lourds et que la vitesse est plus èlevée.

Le systeme de traction a courant continu, tel qu'il vient d'être décrit, fonctionne bien au point de vue mécanique, mais il prêsente deux défauts très graves, d'abord son prix élevé de premier établissement et d'exploitation, dù à la nécessité d'installer le long des voies un grand nombre de sous-stations pour alimenter le troisième rail, en second lieu et principalement le mauvais rendement de l'ensemble

(-) Par l'expression "traction électrique", jentends parler des procédés de traction électrique qu comportent le transport de l'énergie electrique depuss une station centrale jusquaux véhicules moteurs à l'aide de conducteurs sur lesquels s'appuiont un ou plusieurs contacts girsants. Je lassse de côté le procédé de traction électrique par accumulateurs portés par le véhicule moteur, parceque ce système. qui présente toutes sortes d'inconvements, est le plus coùteux de tous. de l'installation. Ce mauvais rendement tient d'une part à la perte considérable d'énergie dans les conducteur's de distribution, causée par les énormes intensités qu'on est obligé d'employer, et d'autre part à la perte d'énergie dans les résistances de démarrage et de réglage de la vitesse. Ces dernières pertes sont relativement peu importantes pour les trains directs. mais ellos deviennent considérables pour des trains à arrêts fréquents.

La tension de distribution employée dans les installations de traction à courant continu est ordinairement limitée à 600 volts, parce qu'il est difficile d'établir et surtout d'entr'. tenir des moteurs électriques de traction à courant continu fonctionnant convenablement, et sûrement, à une tension notablement plus élevée.

D'autre part, pour une tension plus haute, on ne peut plus emplnyer un conducteur de grande section placé prèsdu sol, à cause du danger et des difficultés d'isolement, et on est contraint de revenir au conducteur aérien, lequel doil nécessairement avoir une section relativement faible.

Une des installations les plus intéressantes de tractiona courant continu oú il est fait usage d'une tension notable. ment supérieure à 600 volts, est celle du chemin de fer de St-Georges-de-Commiers à La Mure $\left(^{\star}\right)$.

Sur ce chemin de fer, il est fait usage de locomotives munies de 4 moteurs à 600 volts montés en tension.

Le courant est transmis aux locomotives par deux fils aériens, entre lesquels existe une différence de tension de 2.400 volts, et qui sont en contact avec deux trolleys dis. tincts connectés chacun avec une des extrémités du groupe des quatre moteurs. Le milieu du groupe formé par les qualre moteurs est relié à la terre, c'est-à-dire à la voie, de sorte que la différeñce de tension maxima du système, par rapport à la terre, est égale à 1.200 volts seulement.

Ce procédé a l'avantage de diminuer les difficultés d'iso. lement des moteurs, mais, par contre, il a l'inconvénient grave d'exiger l'emploi de deux fils aériens distincts, ce qui complique beaucoup l'établissement des lignes de prise de courant au droit des aiguillages, croisements, etc.

Il permet l'alimentation, au moyen d'une seule usine génératrice, d'une petite ligne de longueur et de trafic mo. dérés, mais il ne résout nullement le problème général de l'application de la traction électrique sur les grandes lignes; d'autre part, l'utilisation de l'énergie électrique recueillie par les locomotives se fait d'une manière aussi défectueuse que dans le système ordinaire lors des démar. rages et variations de vitesse.

\section{EMPLOI DES COURANTS TRIPHASÉS}

Pour transporter économiquement l'énergie électrique de grandes distances, il est nécessaire d'employer des tensions aussi élevées que possible.

Partant de cette idée, d'importantes sociétés de constructions électriques ont cherché à appliquer à la traction la transmission par courants alternatifs triphasés qui se prëte très bien à l'emploi des tensions élevées.

Ce système de traction électrique, encore assez pell répandu, et dont les applications les plus importantes ont. été faites sur la ligne de la Valteline, en Italie, et à la traversée du tunnel du Simplon, comporte l'emploi de trois conducteurs, dont deux sont aériens et isolés, le troisième. conducteur étant constitué par la voie.

Les moteurs montés sur les véhicules, ou les locomolives, sont triphasés du type dit asynchrone.

Ces moteurs peuvent, sans difficultés spéciales, étre construits pour fonctionner à une tension élevée, de sorté qu'll est possible d'opérer la transmission de l'énergie all moyen de conducteurs de sections réduiles, et par suite d'alimenter une ligne de grande longueur par une seule

(") Vour La Houille Blanche, novembre 1903. 
station génératrice, sans faire usage de sous-stations comme dans le système à courant continu.

routelois, si on peut se passer de sous-stations proprement dites, il est nécessaire d'établir le long de la ligne de nombreux postes de transformateurs statiques, de manière a maintenir à un taux convenable la tension sur les fils de prise de courant.

Les moteur's triphasés, en effel, contrairement à ce qui se passe pour les moteurs à courant conlinu, ne peuvent fonctionner d'une façon satisfaisante que si la tension des courants qui les alimentent reste comprise dans des limites assez étroites; si cette tension baisse, le couple diminue considérablement.

1 a vitesse de rotation des moteurs triphasés est prati_ quement déterminée par le nombre d'alternances par ceconde des courants triphasés qui les alimentent, et est ¿ $2 \%$ près environ, indépendante de l'intensité du courant qui les traverse lorsque les rhéostats de démarrage sont hol's circuit.

Il en résulte que les locomotives triphasées ont unevilesse constante malgré les variations du profil, contrai rement à ce qui a lieu dans tous les autres systèmes de traction électriçue, et, comme conséquence, doivent avoir une puissance très supérieure à la puissance moyenne.

C'est un grave inconvénient, car la station centrale qui alimente le chemin de fer subit de ce chef des à-coups considérables et doit, de temps à autre, fournir une puissance beaucoup plus grande que la puissance moyenne.

le prix de premier établissement d'une telle station est for'ément élcvé, et, si la force motrice est produite par la vapeur, lo rendement moyen est nécessairement médiocre.

Pour atténuer l'inconvénient résultant de la constance de la vitesse, on a imaginé divers disposilifs qui, par des modifications dans le groupement des circuits, montage en tandem ou changement dans le nombre des póles, permettent d'obtenir deux vitesses de régime; mais ces vitesses sont elles-mêmes constarites et bien déterminées, et on ne peut passer de l'une à l'autre qu'en faisañt usage de résistances de réglage pendant toute la durée du changement de vilesse, ce qui diminue le rendement.

Dans aucun cas, on ne peut varier d'une façon continue la vitesse de marche suivant la nature du profil, en maintenant le rendement sensiblement constant.

L'un des plus grands inconvénients du système de traction yar courants triphasés est sans contredit l'obligatoin d'employer deux conducteurs aériens de prise de courant ce qui, comme je l'ai déjà fait remarcuer á propos du système de traction à courant continu à deux fils aériens employé sur le chemin de fer de St-Georges-de-Commiers à La Mure, rend très difficile l'établissement des conducteurs au droit des aiguillages, croisements, etc..

Sur une petite ligne où les gares sont peu développées, il est encore possible de s'en tirer, mais la complication du réseau aérien deviendrait invraisemblable dans une grande gare.

\section{EMPloi du Courant alternatif Monophasé}

Convaincus que l'emploi des courants alternatifs triphasés ne saurait conduire à la véritable solution du problème de la traction ólectrique sur les grandes lignes de chemins de fer, un grand nombre d'Ingénieurs ont cherché à utiliser dans ce but les propriètés des moteurs à courant alternatif monophasés à collecteurs, et plusieurs maisons de constructions électriques sont arrivées récemment à créer des types d'un rendement satisfaisant et capables de faire un bon service de traction.

Les avantages théoriques du nouveau système de traction par courant alternatif monophasé sont évidents:

Emploi d'un seul fil aérien de prise de courant avec tension aussi élevée qu'on le désire.

Réduction de la tension sur les véhicules moteurs au moyen de transformateurs statiques, ce qui supprime les difficultés d'isolemient des diver'ses parties des moteurs.

Possibilité de graduer à volonté la tension effective d'alimentation des moteurs, en laisant usage de transformateurs, ou d'auto-transformateurs sectionnés, ou de régulateurs d'induction, ce qui permet de diminuer considérablement les pertes d'énergie électrique lors des démarrages et des variations de vilesse.

Grande souplesse des moteurs qui peuvent fonctionner également bien à des vitesses différentes, et sont, à ce point de vue, comparalles aux moteurs séric à courant continu.

Plusieul's applications du système monophasé, avec diverses variantes, ont été faites recemment aux EtatsUnis et en Europe sur des lignes secondaires qui, au point de vue des puissances mises en jeu sur les véhicules moteurs, sont plutôt comparables à de grands tramways interurbains qu'à de véritables chemins de ler.

Le fonctionnement pratique de ces installations paralt satisfarsant, mais, on n'a pas encore publié do renseignements précis au sujet des rendements réels en service sur lesquels on peut compter, toules pertes déduites.

Encouragés par ces premiers résultats favorables, plusieurs constructeurs ont pensé que la solution du problème de la traction électrique sur les grands chemins de fer serait donnée par l'emploi des moteurs monophasés, et ont orienté résolument leurs recherches daus cette nouvelle direction.

Parmi eux, il convient de citer en premiere ligne la Société américaine Westinghouse qui a tcruiné,en mai 1905, la construction dune grosse locomotive ćloctique monophasée qu'elle a montrée aux Mombres du Congrès des Chemins de fer sur les voies des atelier's de Pittsburg.

Cette locomotive, destinée a la traction des trains de marchandises, a une puissance de 1350 chevaux.

Elle est composée de deux trucks accouplés montés chacun sur trois essieux, tous moteurs, soit six essieux moteurs en tout, et pése 123 tonnes métriques environ.

Chacun de ces essieux est muni de roucs de $1 \mathrm{~m} .524$ et actionné, au moyen d'une iransmission à engrenages, par un moteur monophasé refroidi par ventilation artificielle, d'une puissance de 225 chevaux.

En examinant la locomotive ci-dessus, on constate qu'on a utilisé d'une façon complète tout l'espace dont on pouvait disposer entre les roues, et quil aurait été impossible de loger des moteurs plus volumineux sur los essieux considérés.

Plus récemment, la Compagnie Westinghouse a entrepris l'électrification dune partie de la ligne du Chemin de lér de New-York, Nerv-Haven et Hartford au moyen de locomotives électriques monophacées, directement alimentées par un fil aérien à la tension de 11000 volts.

Les nouvelles locomotives, destinées a la traction des trains de voyageurs, pèsent environ 78 tonnes métriques el sont montées sur deux bogies moteurs munis de roues de $1 \mathrm{~m} .575$ de diamètre.

Sur chaque essieu est enfilé un moteur monophasé à action directe, refroidi par ventilation artificielle, dune puissance normale maximum de 250 chevaux, ce qui donne 1000 chevaux comme puissance normale maximum de la locomotive.

Des deux exemples ci-dessus, il parait ressortir d'unc manière évidente que, pour le moment du moins, il est impossible de loger un moteur monophasé de plus de 250 chevaux sur un essieu muni de roues atteignant $1 \mathrm{~m} .575$ de diamètre. La puissance maxima d'un moteur. de ce type serait naturellement moindre pour un diamètre de roue plus faible.

Les locomotives à courant continu construites par la General Electric Company, de Schenectady, pour le chemin de fer du New-York Central and Irudson River, sont munies de 4 essieux moteurs, comme celles du chemin de fer de 
New-York, New-Haven et Hartford, el ces essieux, qui sont munis de roues de $1 \mathrm{~m}$. 117 seulement, sont actionnés chacun par un moteur à courant continu à action directe, d'une puissance normale maximum de 550 chevaux, de solte que les locomotives électriques du New-Yorli Central, qui ont un poids de 90 tonnes métriques, ont une puissance normale maximum de 2200 chevaux.

On voit par ce qui précède qu'on a pu loger un moteur à courant continu non ventilé de 550 chevaux sur un essieu muni de roues de $1 \mathrm{~m}$. 117 seulement, tandis qu'on n'a pu loger qu'un moteur de 250 chevaux à courant alternatif monophasé sur un essieu muni de roues de $1 \mathrm{~m} .575$, et encore à la condition de le refroidir par ventilation artificielle.

On ne pourrait arriver à construire de très puissantes locomotives à moteurs monophasés qu'en employant un grand nombre d'essieux moteurs, 8 au moins pour une locomotive de 2000 chevaux, d'où il résulte que ce système, indépendamment de toute autre considération, ne peut conduire à une solution satisfaisante du protlème de la traction électrique sur les Chemins de fer, lorsqu'il est nécessaire de remorquer des trains lourds à grande ritesse sur une ligne à profil difficile.

J'ai laissé complètement de côté la question, pourtant fort importante, du rendement total qu'aurait une grande installation de traction à moteurs monophasés car, ainsi que je l'ai déjà fait remarquer, on n'a encore rien 'publié de précis à ce sujet.

\section{Fimplor simultané du Courant alternatif Monophasé ET DU CoURant CONTINU}

Les moteurs à courant continu sont ceux qui conviennent le mieux lorsqu'il est nécessaire d'avoir sur chaque essieu une puissance élevée, et, d'autre part, en employant du courant alternatif monophasé à haute tension, on peut transmettre facilement à de grandes distances une quantité considérable d'énergie.

Partant de ces données, on a cherché à établir des locomotives électriques dont les essieux seraient actionnés par des moteurs à courant continu et auxquelles l'énergie électrique serait amenée depuls la station centrale sous forme de courant alternatif monophasé au moyen d'un seul conducteur aérien isolé, le deuxième conducteur étant constıtué par les rails de la voie.

Les ateliers d'Erlikon ont construit sur ce principe une locomotive électrique d'essai, d'une puissance de 500 chevaux, qui recueille sur un fil aérien isolé du courant alternatif monophasé à la tension de 14000 volts environ.

Ce courant actionne un moteur asynchrone qui fait toumer une dynamo génératrice à courant continu, et le courant ainsi obtenu est envoyé dans les moteurs des essieux.

La dynamo génératrice est à excitation indépendante et variable, et peut produire une tension variable à volonté depuis zéro jusqu'à 600 volts.

Le fonctionnement de l'ensemble est satisfaisant, en ce sens que le démarrage se fait avec la plus grande facilıté, sans à-coups et sans perte d'énergie dans des résistances.

Il en est naturellement de même du réglage de la vitesse.

Bien qu'il y ait une double transformation sur la locomotive de l'énergie électrique en travail mécanique, et vice versa, le rendement du système est certainement meilleur que dans une installation ordinaire à courant continu, et le rapport moyen entre la puissance mécanique mesurée à la jante des roues motrices de la locomotive et l'énergie électrique produite dans la station contrale sous forme de courant monophasé à haute tension peut certainement atteindre $64 \%$.

Malgré les avantages ci-dessus, ce système ne paraît pas constituer la véritable solution du problème général de la traction électrique, parce que le poids, et surtout l'encom. brement des moteurs générateurs, sont nécessairement très élevés, ce qui rendrait bien difficile l'établissement des très puissantes locomotives qui sont, ou qui vont, devenir indispensables sur les chemins de fer, en raison de laugmentation continue du poids et de la vitesse des trains; mais, le principal inconvénient du systeme do transformation du courant alternatif en courant continu par moteur générateur est le manque de stabilité de ce dernier appareil.

Si en effet, pour une cause quelconque, le courant alter. natif à haute tension cesse de parvenir à la locomotive, même pendant un temps très court, le moteur, freiné par la dynamo génératrice $\dot{a}$ courant continu qu'il conduit, se ralentit instantanément et s'arrête presqu'aussitôt, même si le courant à haute tension lui est ensuite rendu.

Aucun des systèmes électriques connus ne nous ayant paru capable de lutter avec la vapeur, sauf dans des cas spéciaux, comme la traction sur de très fortes rampes ou sous de longs tunnels, nous avons été conduits, à propos d'une application particulière de la traction électrique pro. jetée sur une des lignes de la Compagnie P. L.-M., à étudier un nouveau système dont je vais exposer brièvement les dispositions caractéristiques.

Aucune décision n'a encore été prise au sujet de lapplication en question, mais la Compagnie P.-L.-M. a lait procéder à des essais préliminaires très importants en vue de vérifier l'exactitude des principes qui servent de base au nouveau système, et de déterminer par expérience les coefficients qu'il conviendrait d'employer lors de la construction des locomotives électriques.

Nous avons vu tout à l'heure que, parmi tous les sys. tèmes de traction examinés, celui qui comporte l'emploi du courant alternatif monophasé à haute tension pour le transport de l'énergie électrique depuis la station centrale jusquaux locomotives, puis la transformation du courant monophasé en courant continu à tension variable pour actionner les moteurs des essieux, est le seul qui allie l'économie dans le transport de l'énergie électrique à la facilité et à l'économie de l'emploi de cette énergie sur les locomotives; pour qu'il soit pratique, il convient d'opérer la transformation du courant monophasé en courant continu au moyen d'appareils ayant une grande stabilité de fonctionnement, et un volume suffisamment réduit pour pouvoir être installés sur les locomotives.

C'est ce problème que j'ai résolu, grâce à l'emploi d'un nouvel appareil, le Redresseur-Régulateur, que j'ai étudié en collaboration avec F. Ferrand, Ingénieur du Service Central du Matériel de la Compagnie P.-L.-M.

Ce redresseur, dans la description duquel je nè puis entrer ici, est un appareil composé de pièces tournantes et de pièces fixes,qui reçoit l'énergie électrique sous forme de courant alternatif monophasé et la restitue sous forme de courant continu, sans qu'il ait été nécessaire de passer par ta double transformation dénergie électrique en travall mécanique, puis de travail mécanique en énergie éler. trique, comme cela a lieu dans les moteurs générateurs (").

Un faible travail mécanique est cependant indispensable en pratique pour faire fonctionner l'appareil, mais seulement pour vaincre les frottements des pièces en mouvement sul' les pièces fixes, ainsi que la résistance de l'air, et ce travail est absolument indépendant de la puissance du courant redressé que l'on recueille; il est le même à vide, à pleine charge ou en surcharge, et la partie tournante du redresseur, une fois lancée, ne se ralentit que lentement, en cas d'interruption du courant alternatif, parce que sa force vive

(*) Dans un prochain numéro, nous donnerons la description de l'ap* carenl de MM. Auvert et Ferrand. 
est considérable par rapport â l'effort retardateur provenant des résistances passives, les seules en jeu.

Il en résulte en pratique une très grande stabilité.

Ce redresseur est appelé aussi régulateur, parce qu'il permet, non seulement de transformer du courant alternatif en courant continu, mais aussi de régler à volonté la tonsion de ce courant continu tout en laissant constante la tension du courant alternatif reçu. Ce réglage se fait depuis zéro jusqu'au voltage maximum, sans perte d'énergie, par simple modification du calage des frotteurs servant à recueillir le courant continu sur la partie tournante de l'appareil.

Un appareil d'essai d'une puissance effective de 500 kilowatts a été construit, à la fin de l'année 1904, pour le compte de la Compagnie P.-L.-M., dans les ateliers Schneider et $\mathrm{C}^{1 e}$, à Champagne-sur-Seine, et a pu être soumis à une première série d'expériences et de mesures; en mai et avril 1905, dans la station centrale du Chemin de fer Métropolitain à Bercy, où le courant alternatif dont nous avions besoin a été mis à notre disposition.

Ces premiers essais, dont les résultats ont élé exposés en détail dans la Revue Générale des Chemins de fer et Iramuays (no doctobre 1905), ont permis de constater que le redressement et le réglage du courant se faisaient dans d'excellentes conditions et que la stabilité de l'appareil était parfaite.

La commutation était très satisfaisante et le rendement total, constaté par des mesures précises faites avec des appareils enregistreurs, dépassait, toutes pertes comprises, $88 \%$ pour un débit de $400 \mathrm{kw} ., 86 \%$ pour un débit de $200 \mathrm{kw}$, et $79 \%$ pour un débit de $100 \mathrm{kw}$.

Le rendement était d'ailleurs à peu près indépendant de la tension du courant continu fourni par l'appareil, ce qui montre que le réglage de la tension se faisait sans perte sensible d'énergie.

Une deuxième série d'essais vient d'être faite récemment avec le redresseur-régulateur par la Société d'Electricité Alioth, dans ses atelier's de Múnchenstein, près Bâle, en vue de compléter les essais que nous avions faits à Paris en 1905 et d'élucider certains points spéciaux qui avaient été primitivement laissés de côté.

On a vérifié ainsi qu'on pouvait sans aucune difficulté alimenter avec du courant redressé des moteurs à excitatation séparée, et qu'on pouvait aussi, à l'aide du redresseur-régulateur, transformer du courant continu à tension valiable en courant alternatif à tension constante.

Il résulte de ce qui précède qua, grâce à l'emploi des redresseurs-régulateurs. un pourra tacilement construire des locomotives électriques dans lesquelles la dépense d'énergie électrique será exactement proportionnée à la production du travail mécanique au moment des démarrages, et pendant la marche normale, et qui jouiront aussi de la propriété précieuse de restituer au réseau, sous forme de courant alternatif, l'énergie disponible correspondant à la diminution de force vive lors des ralentissements, ou au travail de la gravité pendant la descente des pentes.

En raison de l'extrême modérabilité des redresseursrégulateurs, on pourra régler la vitesse sur les pentes et ralentir les trains presque jusqu'à l'arrêt complet, sans faire usage des freins mécaniques.

En lésumé, en combinant les propriétés du courant continu avec celles du courant alternatif, il est aujourd'hui possible de construire des locomotives électriques d'une puissance considérable, plus grande que celle des plus fortes locomotives à vapeur; avec ces nouvelles locomotives, le coút de la traction électrique ne serait, dans beaucoup de cas, pas plus élevé que le coût de la traction à vapeur sur les profils faciles, et sur les profils accidentés, grâce à la récupération, il deviendrait très nettement inférieur.

\section{TRACTION ÉLECTRIQUE DES BATEAUX}

Rapport de M. Léon Gérard

ingénieur, ancien président de la Société Belge des Electrictens.

\section{HISTORIQUE}

L'évolution des moyens de transports est un des faits les plus saillants de l'histoire contemporaine.

Cette évolulion s'est opérée d'une manière très curjeuse, car elle a tour à tour fait abandonner et reprendre les divers Imodes usuels pour les perfectionner ensuite et les déveop per.

Dans les pays les plus industriels, la locomotive, aussitôt après son adoption, a fait complètement abandonner la route de terre, et le désir de limiter la concurrence des moyens de transport a poussé les puissantes compagnies de railways à accaparer les canaux pour les réduire à l'impuissance ; c'est l'histoire des canaux houillier's alimentant les ports anglais au début du XIX siècle.

La France a eu l heureuse fortune de n'avoir jamais abandonné ses admirables routes. Elle en est récompensće par l'essor si remarquable de sa grande industrie nationale de l'automobile.

Par un singulier retour des lois du progrès, toutes les perfections de la mécanique s'appliquent en ce moment à remettre en pleine activité le transport rapide sur route et bientôt sans doute le transport lourd.

Cette évolution récente est d'autant plus remarquable que l'on considérait, il n'y a pas vingt ans, le transport sul' route comme ne présentant qu'un mode arriéré de locomotion destiné à tomber en complète désuétude.

Le même phénomène s'est produit en matière de navigation intérieure. Là, encore, la France jouit à l'heure actuolle du remarquable avantage d'avoir sans cesse entretenu et perfectionné ses voies navigables, et, après une période d'erreur économique qui avait presque livré l'exploitation de ces voles a leur victorieux concurrent, le chemin de fer, on vit successivement se produire l'abolition des péages, la régularisation des grandes rivières, l'approfondissement et la systematisation d'un admirable réseau de canaux dont la législature a décidé, il y a quelques années, l'extension d'aplès un plan grandiose.

\section{IMPORTANCE ÉCONOMIQUE}

En 1905 , le trafic des quelques rivières canalisées françaises atteignait plus de 600 millions de tonnes kilomètres et 1470 kilomètres de canaux proprement dits assuraient à eux seuls un trafic de 2 milliards de tonnes kilométriques. Ces chiffres sont relatils à une petite partie seulement du réseau des canaux trançais, dont l'utilisation économique généralisée dépendra de divers facteurs, parmi lesquels le prix du halage et la vitesse sont des éléments essentiels. La France possédait, en 1904,8 325 kilómètres de rivières, canalisées en 4930 kilomètres de canaux.

Afin de se faire une idée comparative de l'importance économique des transports par eau, le fret moyen par eau pour les marchandises pondéreuses est, en France, le quart du prix du transport par rail.

En Allemagne, ce chiffre est du sixième. En Angleterre le transport par eau est dix fois moins cher et il est, en Belgique et en Amérique, six fois moins couteux que le transport par rail.

L'ensemble du Irafic par eau en France, que l'on peut estimer grossièrement à plus de 4 milliards de tonnes kilométriques, économise annuellement à l'industrie française plus de 96 millions de francs sur les dépenses de transport.

Les raisons techniques de cette formidable supériorité économique des transports par eau résultent de la réduction des efforts de traction, de la grandeur des unités de transport. L'unité moyenne est en France de 350 tonnes, et ce chiffre tend à grandir, et la barque type de navigation intérieure atteindra probablement 600 tonnes. 